\title{
Espacio biológico. Parte I: La inserción diente-encía
}

\author{
DELGADO PICHEL, A. * \\ INAREJOS MONTESINOS, P.* \\ HERRERO CLIMENT, M. **
}

Delgado Pichel A, Inarejos Montesinos P, Herrero Climent M. Espacio biológico. Parte I: La inserción diente-encía. Av Periodon Implantol. 2001; 13, 2: 101-108.

\begin{abstract}
RESUMEN
Se denomina espacio biológico a la unión dentogingival, que está constituida por el epitelio de unión y el tejido conectivo de inserción de la encía. Cuando se habla de espacio biológico no sólo se debe pensar en la longitud de la inserción gingival, sino que se debe relacionar con el grosor de la encía, el biotipo periodontal y la profundidad del surco gingival, puesto que todos estos parámetros se integran, y deben ser tenidos en cuenta para comprender de manera exacta la morfología del tejido gingival supracrestal. La variabilidad de dimensiones de los componentes epitelial y conectivo que existe entre individuos, e incluso dentro del mismo individuo, es otro factor que debe ser considerado. Una vez que se ha invadido la unión dentogingival, el tipo de manifestación clínica que se produce va a ser distinta según los casos, ya que no hay que olvidar que la respuesta está relacionada con la susceptibilidad del paciente frente a la enfermedad periodontal, además de otros factores que se enumerarán.
\end{abstract}

Y cuando se ha invadido el espacio biológico con sintomatología, ¿qué se puede hacer? La parte final esbozará las opciones terapéuticas disponibles frente a estas situaciones.

\section{PALABRAS CLAVE}

Espacio biológico; anchura biológica; biotipo periodontal; alargamiento de la corona dentaria.

\section{INTRODUCCIÓN}

Se denomina espacio biológico a la unión dentogingival, que ha sido descrita como una unidad funcional, compuesta por el tejido conectivo de inserción de la encía y el epitelio de unión (1). (Figura 1)

La importancia de esta estructura radica en las consecuencias que se pueden derivar de su invasión, que como se verá más adelante puede inducir retracción gingival, pérdida ósea, hiperplasia gingival, etc., todo ello con unas graves consecuencias desde el punto de vista de la salud periodontal como de la estética gingival.

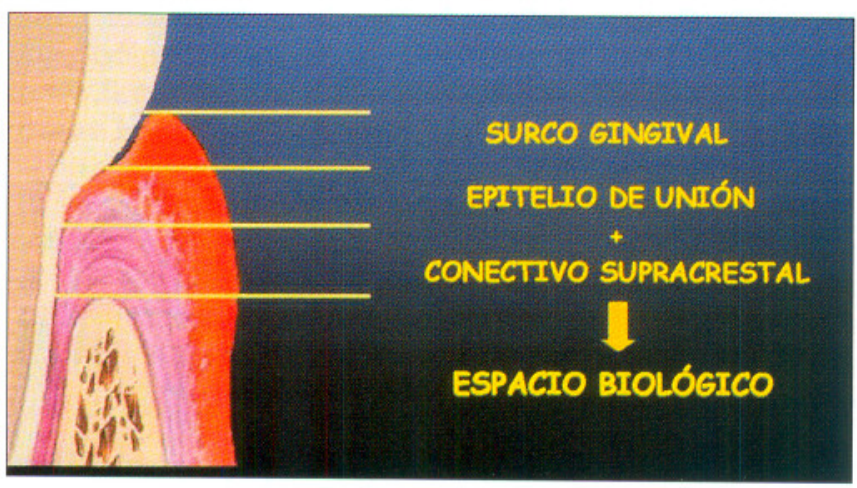

Fig. 1: Esquema del tejido gingival supracrestal.

\footnotetext{
* Odontólogo. Práctica privada.

** Médico-odontólogo. Práctica privada.
} 
Si un tratamiento restaurador requiere una preparación dentaria intracrevicular, se debe estudiar el caso de forma individualizada para elaborar un correcto plan de tratamiento, analizando si se va a necesitar algún tratamiento periodontal u ortodóncico previo para alargar la corona dentaria; se deberá determinar cuanto es posible insinuarse en el surco gingival, porque cuando se habla del espacio biológico se tiene que tener presente que es una característica morfológica gingival totalmente personal y propia de cada paciente, y por lo tanto los valores promedio obtenidos, por ejemplo, por Gargiulo (2) no son aplicables.

Si por ejemplo esa necesidad de preparación intracrevicular se debe a motivos estéticos, y a consecuencia de una incorrecta planificación se produce una invasión del espacio biológico, el resultado a cortomedio plazo tiene un grave impacto en la estética gingival y evidentemente en la estética general del caso, sin olvidar la afectación de la salud periodontal.

Por consiguiente se debe explorar minuciosamente para diagnosticar y planificar cada caso en particular.

\section{ANTECEDENTES}

Gottlieb (1921), y Orban y Mueller (1929), en estudios iniciales del epitelio, describieron una inserción del mismo al diente, presentando un concepto innovador que en ese momento no fue universalmente aceptado. Orban y cols., más tarde (1956) demostraron esto en cortes histológicos al microscopio óptico. Posteriormente, Sicher (1959) describió una unión dentogingival alrededor del diente que comprendía dos partes, una inserción de tejido fibroso y una inserción de epitelio (3).

En 1962, Cohen definió la "anchura biológica" (es más apropiado denominarlo "espacio biológico", ya que hace referencia a la dimensión longitudinal, y no transversal (4)) del tejido gingival supracrestal como aquellos elementos del epitelio de unión y tejido conjuntivo del complejo dentogingival que ocupan el espacio comprendido entre la base del surco gingival y la cresta alveolar. Basándose en Gargiulo y cols., la dimensión total del tejido gingival supracrestal (TGS) es de 2,04 mm (2,73 mm si se tiene en cuenta el surco gingival).

Basándose en estas dimensiones, Ingber y cols, afirmaron que se debía resecar en los alargamientos de corona dentaria quirúrgicos hueso suficiente como para permitir $3 \mathrm{~mm}$ de estructura dental sólida encima de la cresta del hueso; Rosenberg y cols. preconizaron $4 \mathrm{~mm}$ de exposición dental (5).

\section{RECUERDO HISTOLÓGICO}

El tejido conectivo supracrestal está formado por fibroblastos (5\%); células de los vasos sanguíneos, linfáticos, terminaciones nerviosas y matriz (35\%) y fibras colágenas tipo I $(60 \%)$, que se organizan en haces: grupo gingivodental (FDG), periostiodental (FDP), circular (FC), alveologingival y transeptal (FT), que para algunos forman ya parte del periodonto (6) (Figura 2).
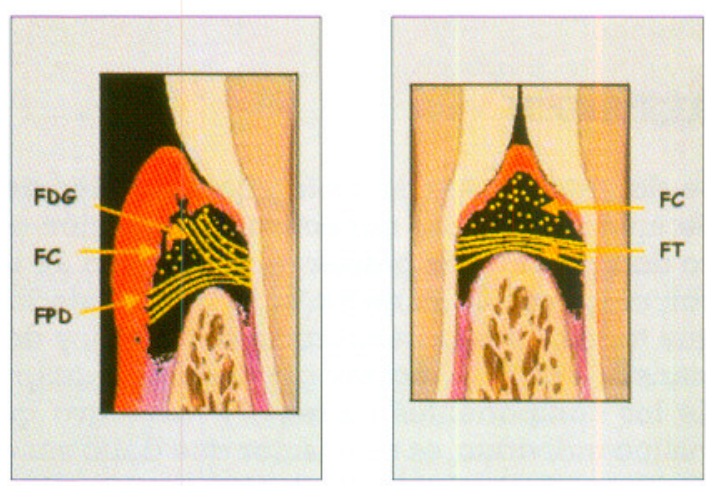

Fig. 2: Haces de colágeno del tejido conectivo.

El epitelio de unión se organiza como un epitelio no queratinizado, escamoso y estratificado. Está formado por queratinocitos (capa basal y estrato espinoso) y otras células (no queratinocitos o células claras) como son melaninocitos, cels. Merckel, linfocitos T y B, macrófagos y PMns. Las céls. de Langerhans, a diferencia del epitelio bucal y del sulcular, tal vez no estén presentes (1).

Es más grueso en su zona más coronal (15-20 capas celulares), que en su basal, donde se producen las mitosis. Desde ahí las células migran hacia el surco gingival (el suelo de la hendidura está constituido por los elementos superiores del epitelio de unión) (6).

La adherencia epitelial real al diente es efectuada por los hemidesmosomas y la lámina basal interna, que se adhieren a la superficie del diente (esmalte, cemento) e incluso a la superficie de los implantes de titanio. La adhesión con el tejido conectivo gingival se realiza por medio de la lámina basal externa (1). (Figura 3)

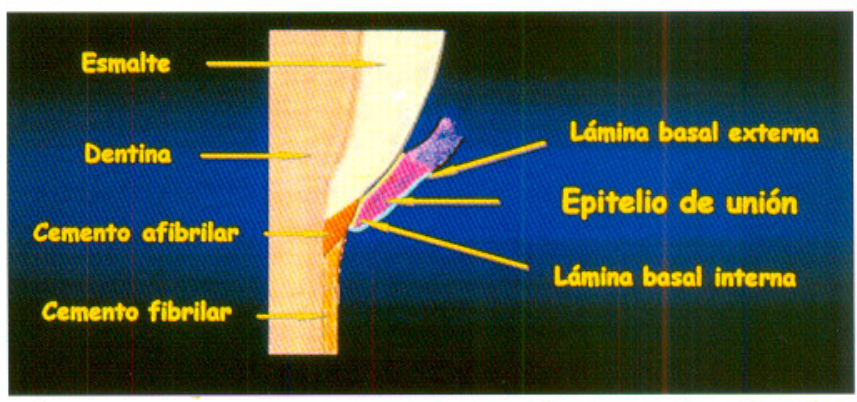

Fig. 3: Esquema del epitelio de unión. 
La riqueza de desmosomas es menor que en el epitelio bucal y que en el sulcular, lo cual le hace más permeable al paso de moléculas y otras células transeúntes. Por ello la adhesión del epitelio de unión al diente se puede interrumpir con relativa facilidad. Cuando ocurre, la cohesión entre las células epiteliales y las otras capas de tejido de la unidad dentogingival se debilita, y se propiciará un cambio inflamatorio, disponiendo el escenario para la destrucción periodontal (5).

El epitelio de unión tiene su origen embriológico en el epitelio reducido del esmalte; en el momento en que se produce la erupción dentaria, se va sustituyendo progresivamente

el epitelio reducido por el de unión, avanzando dicho cambio apicalmente hasta que el diente alcanza su posición definitiva en la arcada (6). (Figura 4)

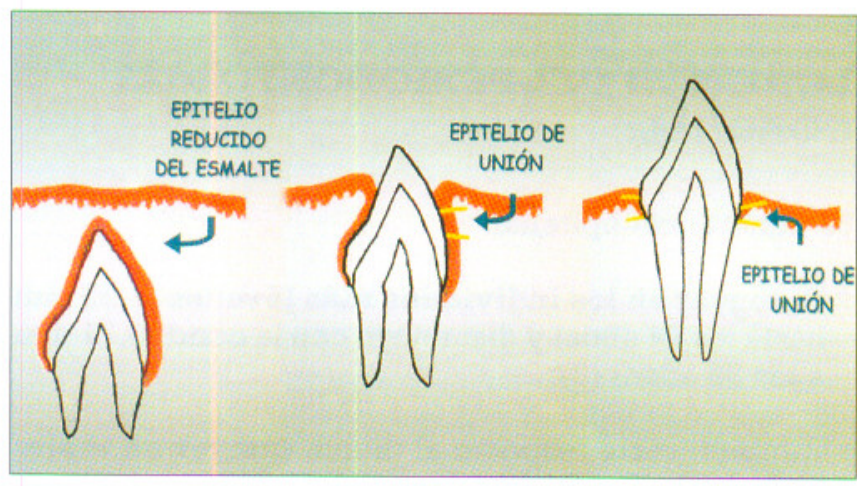

Fig. 4: Formación del epitelio de unión durante la erupción dentaria.

Según el concepto de la erupción ininterrumpida o continua (Gottlieb), la erupción no cesa cuando los dientes encuentran sus antagonistas funcionales sino que persiste toda la vida. Basándose en esto se habla de erupción activa y erupción pasiva:

La erupción activa es la que se produce como compensación a la atricción que tiene lugar a lo largo de la vida, lo cual evita idealmente la disminución de la dimensión vertical. Se acompaña de aposición de cemento a nivel de los ápices y las furcas $(1,7)$.

La erupción pasiva consiste en la migración apical de la encía, con la consiguiente exposición dentaria. Esto hoy día se considera patológico. Se establecen 4 fases en función de la localización de la encía $(1,8)$ :

* Fase 1: la distancia desde encía marginal hasta cresta ósea es de 3,23 mm (de los que 2,43 mm son de espacio biológico), estando la encía marginal y el epitelio de unión en la superficie del esmalte.

* Fase 2: la distancia es de 3,06 mm (2,45 mm de espa- cio biológico); la encía marginal está sobre el esmalte y el epitelio de unión está parcialmente sobre el esmalte y el cemento. La base del surco gingival aún permanece sobre el esmalte.

* Fase 3: la distancia es de 2,41 mm (el espacio biológico es de 1,80 mm) y la encía marginal está en la unión amelocementaria; el epitelio de unión está totalmente sobre el cemento. La base del surco se localiza en la UAC.

* Fase 4: la distancia es de 2,53 mm (1,77 mm de espacio biológico); la encía marginal y el epitelio de unión están sobre el cemento (recesión). La base del surco se localiza sobre el cemento.

También se dan casos en los que el margen gingival se localiza coronalmente a la UAC, lo que se conoce con el nombre de erupción pasiva alterada. Esta situación se puede acompañar de una localización de la cresta ósea normal (1 ó 1,5 mm apical a la UAC) o coronal a su localización normal, situándose sobre la UAC (9).

En la literatura se encuentran presentes dos artículos destacables respecto al estudio de la unión dentogingival, que se basaron en el estudio y medición de múltiples muestras histológicas procedentes de necropsias (Tabla 1):

1. Gargiulo y cols. (1961): 325 superficies dentarias, de edades comprendidas entre los 19 y 50 años, libres de patología periodontal (2).

2. Vacek y cols. (1994): 171 superficies dentarias, de edades comprendidas entre los 54 y 78 años (10).

Entre ambos estudios existen diferencias que se pueden deber a los distintos criterios de selección y análisis de las muestras: la edad de las muestras, la técnica del análisis histológico y la existencia o no de patología periodontal (en el estudio de Gargiulo se descartaron las muestras con patología periodontal) (4).

\begin{tabular}{lrr}
\hline & Gargiulo, 1961 & Vacek, 1994 \\
\hline Surco gingival & $0,69(0,0-5,3)$ & $1,32(0,2-6,0)$ \\
Adherencia epitelial & $0,97(0,08-3,7)$ & $1,14(0,3-3,2)$ \\
Inserción conectiva & $1,07(0,0-6,5)$ & $0,77(0,2-1,8)$ \\
\hline
\end{tabular}

Tabla 1: Resultados obtenidos en dos estudios que analizan la longitud de la inserción dentogingival.

En el estudio de Vacek se registraron las medidas del surco gingival (SUL); el epitelio de unión (EU); el tejido conectivo insertado (TCI) y la pérdida de inserción (PI), que se corresponde con la distancia desde la UAC hasta la zona más coronal del tejido conectivo insertado. 
Los resultados que se obtuvieron fueron:

* No hubo diferencias significativas entre la medida de las distintas superficies dentarias dentro del mismo diente.

* El espacio biológico osciló entre 0,75 y 4,33 mm., por lo tanto no se puede hablar de dimensión ideal en términos generales, ya que la variación entre individuos y entre dientes es muy variable.

* El espacio biológico (la dimensión del TCI y del EU) de los dientes posteriores era significativamente mayor que la de los dientes anteriores. Comparando molares y premolares, sólo la dimensión del TCI fue significativamente mayor (Tabla 2):

* Las superficies dentarias con restauraciones subgingivales tenían significativamente un mayor EU que los dientes no restaurados, pero no existieron diferencias significativas en el TCI, SUL o PI. Cuando se comparó la anchura biológica de los dientes restaurados con los no restaurados, se vio que era mayor en los primeros. No se vio que existieran diferencias en cuanto al tipo de restauración (Tabla 3):

\begin{tabular}{lccc}
\hline & & Medidas (media \pm SD) & Rango \\
\hline EU & Anterior & $1,03 \pm 0,45$ & $0,38-2,48$ \\
& Premolar & $1,20 \pm 0,53$ & $0,32-3,27$ \\
& Molar & $1,22 \pm 0,46$ & $0,44-2,30$ \\
TCI & Anterior & $0,71 \pm 0,24$ & $0,35-1,34$ \\
& Premolar & $0,77 \pm 0,31$ & $0,29-1,84$ \\
& Molar & $0,89 \pm 0,31$ & $0,40-1,77$ \\
PI & Anterior & $3,33 \pm 1,99$ & $0,76-8,73$ \\
& Premolar & $2,73 \pm 1,37$ & $0,87-6,58$ \\
& Molar & $2,76 \pm 1,65$ & $0,60-6,50$ \\
SUL & Anterior & $1,19 \pm 0,89$ & $0,43-6,03$ \\
& Premolar & $1,30 \pm 0,68$ & $0,26-3,24$ \\
& Molar & $1,54 \pm 0,80$ & $0,56-4,04$ \\
\hline
\end{tabular}

Tabla 2: Dimensiones longitudinales de los distintos elementos que intervienen en la inserción dentogingival, analizado por grupos dentarios.

\begin{tabular}{lccc}
\hline & & Medidas (media \pm SD) & Rango \\
\hline EU & Restaurado & $1,32 \pm 0,47$ & $0,69-2,29$ \\
& No restaurado & $1,11 \pm 0,49$ & $0,32-3,27$ \\
TCI & Restaurado & $0,84 \pm 0,26$ & $0,42-1,47$ \\
& No restaurado & $0,76 \pm 0,29$ & $0,29-1,84$ \\
PI & Restaurado & $2,60 \pm 1,53$ & $0,60-8,73$ \\
& No restaurado & $3,01 \pm 1,73$ & $0,74-8,73$ \\
SUL & Restaurado & $1,60 \pm 0,80$ & $0,64-4,04$ \\
& No restaurado & $1,27 \pm 0,79$ & $0,26-6,03$ \\
\hline
\end{tabular}

Tabla 3: Dimensiones longitudinales de los distintos elementos que intervienen en la inserción dentogingival, analizando según posean restauraciones subgingivales o no.

\section{RELACIÓN ENTRE LA LONGITUD, EL GROSOR DEL TGS Y EL BIOTIPO PERIODONTAL}

Anatómicamente es tan importante valorar el periodonto en su dimensión longitudinal, como en su dimensión transversal, en términos de anchura.

La importancia de la longitud radica en que representa unas dimensiones para los componentes conectivo, epitelial y surco gingival, que siendo inviolables, deben considerarse y respetarse al alargar el diente.

La importancia de la anchura se debe a que está íntimamente relacionada con el parámetro longitud. Ignorar el patrón morfológico puede llevar al fracaso (4).

\section{LONGITUD DEL PERIODONTO MÁS CORONAL:}

La adherencia epitelial:

* Es mayor en los individuos más jóvenes (1,35 mm hasta los 24 años) y disminuye con la edad $(0,71 \mathrm{~mm}$ a los 39 años) (2).

* También varía respecto al diente (mayor en molares): $1,03 \mathrm{~mm}$ en incisivos y 1,22 $\mathrm{mm}$ en molares (10)

El surco gingival:

* Es menor en los más jóvenes: $0,8 \mathrm{~mm}$ frente a los 1,7 mm de promedio en adultos (2).

* Varía de forma similar según el diente (mayor en molares): $1,19 \mathrm{~mm}$ en incisivos y $1,54 \mathrm{~mm}$ en molares (10).

La inserción conectiva:

* Es la dimensión menos variable: 0,7 \pm 0,29 mm según Vacek y cols.

Como se ve, en los más jóvenes el surco gingival es menos profundo, la adherencia epitelial es más larga y la cresta ósea está más coronal, siendo la distancia promedio desde la cresta a la UAC menor de $1 \mathrm{~mm}$ antes de los 20 años.

En el adulto, el surco gingival es mayor, la adherencia es más corta y la cresta ósea está más alejada de la UAC, con valor promedio de 2,15 mm. (Figura 5) 


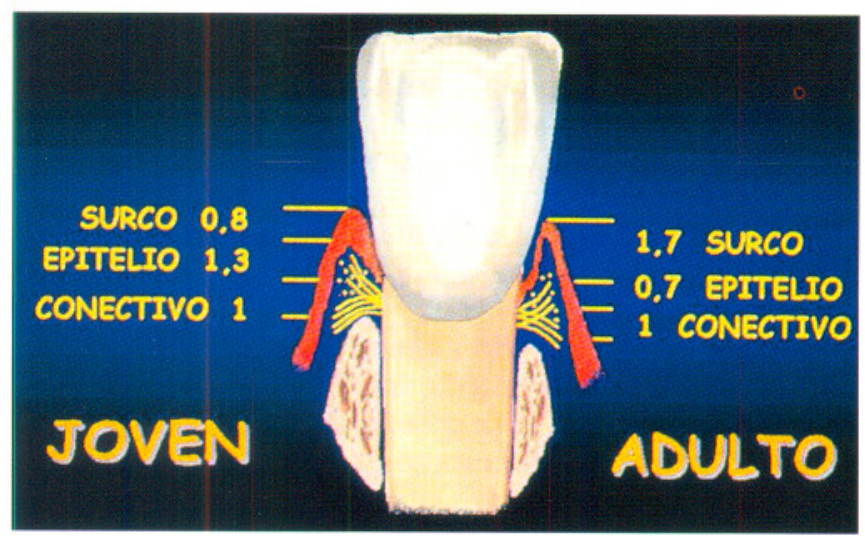

Fig.5: Comparación del TGS en el paciente joven y

El hecho de que la cresta ósea esté más apical en los individuos de mayor edad, en condiciones de salud periodontal, se debe a la erupción activa. En este proceso la cresta sigue al diente hasta cierto límite.

Las dimensiones de la unión dentogingival, con su variabilidad intra e interindividual, están biológicamente determinados; son inviolables (4).

\section{ANCHURA DEL TEJIDO GINGIVAL SUPRACRESTAL (4).}

Referido a espesor de encía. La importancia de esta dimensión es triple:

1. Por la relación entre los parámetros anchura y longitud.

2. Por la relación de la anchura y el biotipo periodontal.

3. Porque la anchura es un condicionante clave del tipo de cicatrización tras la remodelación ósea: la encía fina tiende a la recesión de forma inmediata tras la cirugía, mientras que la ancha tiende a volver a su posición original previa a la cirugía (11).

\section{BIOTIPO PERIODONTAL (4):}

Existe un rango de biotipos periodontales que van desde el denominado biotipo fino hasta el ancho. Las características que les definen son (11):

* Biotipo fino: margen gingival fino y festoneado, con papilas altas.

* Biotipo ancho: margen gingival ancho y poco festoneado.

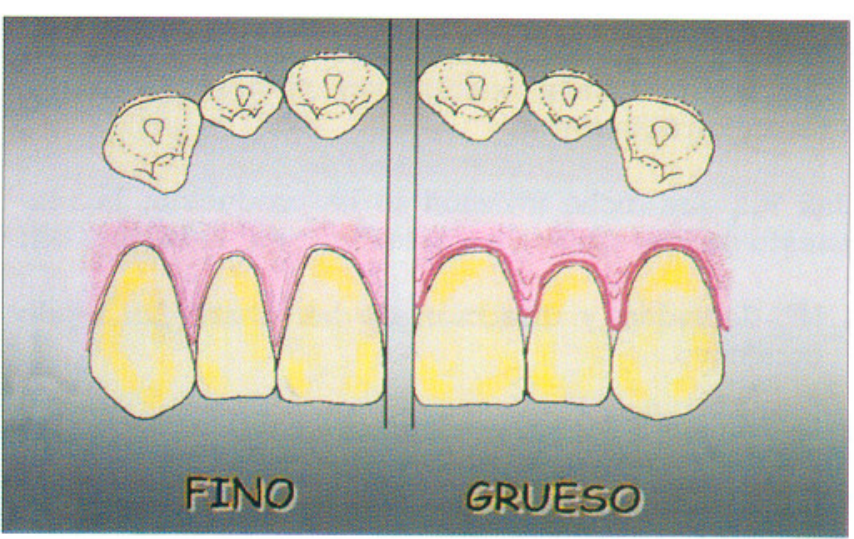

Fig. 6: Periodonto fino frente a periodonto grueso.

También parece existir relación estrecha entre el biotipo periodontal y la anatomía dentaria coronal y radicular (12):

* El periodonto fino se asociaría con coronas largas y cónicas, con puntos de contacto finos. A nivel radicular presentarían contornos convexos prominentes.

* El periodonto ancho lo haría con coronas cortas y cuadradas, con puntos de contacto anchos. La superficie radicular presenta contornos radiculares aplanados. (Figura 6)

Ochsenbein y Ross apreciaron que estas diferencias son también aplicables a la morfología de la cresta ósea subyacente.

Es posible que estas diferencias se reflejen también en la longitud de la unión dentogingival, de forma que el periodonto fino se acompañara de una menor dimensión longitudinal de la unión dentogingival, mientras que la encía más gruesa tuviera una unión más larga. Histológicamente no se ha demostrado, pero en clínica sí ha sido observado; los molares presentan encía y cresta ósea más ancha, y unión dentogingival mayor que los incisivos, con encía y cresta más fina. En la cirugía se puede modificar el parámetro anchura si se quiere modificar la longitud de la unión dentogingival (4).

\section{INVASIÓN DEL ESPACIO BIOLÓGICO:}

Las situaciones en las que se puede provocar una invasión del espacio biológico son las siguientes (13):

\footnotetext{
* Durante el tallado.

* Durante la retracción gingival.

* Durante la toma de impresiones.

* Cementado de restauraciones.
} 
* Restauraciones sobreextendidas.

* Uso de instrumental rotatorio para curetear el surco (8).

* Electrocirugía (8).

Una vez que dicha invasión se ha producido, la respuesta de los tejidos va a estar influenciada por (14):

1. $\mathrm{N}^{\circ}$, densidad y dirección de las fibras del tejido conectivo.

2. La densidad del trabeculado óseo.

3. Localización de los vasos sanguíneos y su emergencia desde la cresta ósea.

4. La interacción inmunológica entre las bacterias y el huésped.

Las alteraciones patológicas que se pueden dar son $(14,15)$ :

1. Pérdida de cresta ósea, desarrollándose una bolsa localizada infraósea.

2. Recesión gingival y pérdida ósea localizada. Rubber y cols. demostraron que existe una mayor susceptibilidad en los casos de crestas finas. La cresta ósea está formada por hueso cortical y una pequeña cantidad de hueso medular. De la medular provienen las células pluripotenciales, las cuales pueden diferenciarse en osteoblastos. Al ser tan escasa, la capacidad de osteogénesis está disminuida, sino eliminada en la cresta ósea, y tras una reabsorción postquirúrgica es difícil la recuperación.

3. Hiperplasia gingival localizada, con mínima pérdida ósea. Es probablemente el cuadro patológico con mejor pronóstico de cara a la longevidad del diente. Se da en áreas de periodonto grueso (interproximal). La estética se ve claramente afectada. Esto se ve a menudo en los casos de erupción pasiva alterada cuando los márgenes de la restauración son realizados subgingivalmente. La resistencia del huésped puede jugar un papel crucial en la respuesta.

\section{Combinaciones de las distintas respuestas.}

No hay que olvidar que la respuesta está relacionada con la susceptibilidad del paciente frente a la enfermedad periodontal (15), por lo tanto no siempre que se invade espacio biológico se producen estos efectos, ya que existen otros factores de iniciación y progresión de la enfermedad periodontal como son la virulencia de la placa y la susceptibilidad del huésped. En estos casos el trauma es reversible para el epitelio y el conectivo, siempre que las condiciones medioambientales sean favorables, produciéndose un nuevo epitelio en 7 - 14 días (13). Anteriormente se pensaba que la invasión de la anchura biológica requería su restauración. Sin embargo, según Ramfjord (20) si una restauración está bien adaptada, la anchura biológica se restablece normalmente sin necesidad de cirugía (16).

\section{¿Qué se puede hacer frente a la invasión del espa- cio biológico?:}

Si la invasión ha causado alteraciones patológicas, las distintas opciones terapéuticas de que se dispone van encaminadas a alargar la corona dentaria, para procurar al TGS el espacio suficiente para una correcta inserción. Estas opciones son:

* Gingivectomía.

* Colgajo de reposición apical.

* Extrusión ortodóncica.

La indicación de una u otra técnica va a depender de distintos factores, como se va a ver a continuación.

La gingivectomía está indicada cuando:

* La cresta ósea está a un nivel adecuado (17).

* La distancia cresta ósea - encía marginal es mayor de $3 \mathrm{~mm}$ (17).

* Hay suficiente encía insertada (17).

* El biotipo es fino (18).

* Se ha mostrado muy útil en caso de sonrisa gingival (8), si esta se debe a erupción pasiva alterada.

* CONTRAINDICADA cuando existe riesgo de exposición radicular (8).

El colgajo de reposición apical (CRA) está indicado cuando (18):

* Se pretende exponer superficie radicular (como mínimo en 3 dientes).

* Biotipo fino o ancho. Este último va a poder ser modificado a un biotipo fino y festoneado.

* Indicado cuando se pretende el alargamiento de múltiples coronas dentarias en un sector.

* CONTRAINDICADO si no existe suficiente inserción periodontal.

* También CONTRAINDICADO cuando sólo se pretende alargar un diente, especialmente en el grupo anterior. 
La extrusión ortodóncica está indicada cuando (19):

* Se desea intervenir en un único diente.

* La longitud de la raíz dentro del hueso debe ser adecuada, puesto que tras la extrusión algo de inserción se habrá sacrificado y la proporción corona-raíz debe seguir siendo correcta para permitir su posterior restauración.

* El diente debe estar endodonciado perfectamente, sin patología periapical.

* El paciente debe estar motivado para aceptar las características del tratamiento: aparatología, varias citas...

La ventaja que presenta esta técnica es que no se abre un espacio interproximal, ni se pierde papila por lo que además no se corre el riesgo de inducir alteraciones fonéticas.

En función de la intensidad de las fuerzas aplicadas, se habla de erupción dentaria lenta o rápida (18) (Tabla 4):

\section{CONCLUSIONES}

Se debe tener un concepto global de lo que es el espacio biológico: no sólo se debe pensar en la longitud, ya que se debe relacionar con el grosor de la encía, el biotipo periodontal y la profundidad del surco gingival, puesto que todos estos parámetros se interrelacionan.
La morfología gingival es una característica propia de cada paciente, y por lo tanto no se puede generalizar y aplicar valores estándar en base a los cuales desarrollar un plan de tratamiento.

Cuando se invade la inserción gingival generalmente se produce una alteración patológica a ese nivel con graves repercusiones. Pero no hay que olvidar que la respuesta está relacionada con la susceptibilidad del paciente frente a la enfermedad periodontal, de forma que no todos los pacientes responden de la misma manera .

Dentro de la planificación de cada caso se debe valorar la necesidad de tratamiento periodontal y/o ortodóncico para prevenir la invasión del espacio biológi$\mathrm{co}$, para corregir dicha invasión si esta ya se ha producido e incluso para tratar casos con finalidades estéticas (por ejemplo corrección de algunos casos de sonrisa gingival).

\section{KEY WORDS}

Width biological, periodontal biotype.

\section{ABSTRACT}

Biological width is known as the dentogingival unit formed by the junction of the epithelium and the connec-

\section{Erupción dentaria RÁPIDA}

Aplicación de fuerzas de mayor intensidad.

Movimiento sólo dentario.

Durante extrusión requiere fibrotomía cada 7-10 días.

\section{Erupción dentaria LENTA}

Aplicación de fuerzas moderadas.

Movimiento de diente y periodonto.

Necesita cirugía posterior en el diente extruído (CRA) para recontorneado óseo.

Indicaciones:

- Nivelar y alinear encía marginal.

- Disminuir la profundidad de las bolsas periodontales cuando existen defectos angulares.

- Alargamiento coronario en zonas donde la remoción ósea de dientes adyacentes deba ser evitada.

- Dientes fracturados (raíz mayor $14 \mathrm{~mm}$ pára correcta proporción corona-raíz) (8).

\section{Contraindicaciones:}

- Si no hubiera suficiente anclaje para la tracción ortodóntica.

- Alargamiento coronario en zonas donde es importante mantener la localización del magen gingival de los clientes adyacentes inalterados.

Tabla 4: Comparación extrusión ortodóncica rápida / extrusión ortodóncica lenta. 
tive tissue attachement. Biological width does not only refer to the length of the gingival insertion, but also to the gingival thickness, the type of periodontal tissues and the depth of the gingival sulcus, because all these components are part of a unit and should be considered as such. Another factor to be considered is the dimensional range of the epithelial and connective tissue components between individuals and in a given individual. Once the dentogingival unit is invaded, different clinical processes can take place regarding the host response to periodontal disease among other different factors that we will expose.

In the event that the biological width has been invaded, and symptoms have appeared, what can be done? At the end and in a nutshell, the reader will find a brief introduction to the different treatment options available for these patients.

\section{CORRESPONDENCIA:}

Alvaro Delgado Pichel

Colegiata, 5 - bajo 3

28012 Madrid

\section{BIBLIOGRAFÍA:}

1. Carranza, Newman. Periodontología clínica. Editorial Mc-Graw Hill Interamericana. $8^{\circ}$ Edición. 1998. Capítulo 1: La encía: $14-32$.

2. Gargiulo AW, Wentz FM, Orban B. Dimensions and relations of the dentogingival junction in humans. J Periodontol 1961; 32: 261 - 267 .

3. David L. Cochran, Joachin S. Hermann, Robert K. Schenk, Frank L. Higginbottom and Daniel Buser. Biologic width around titanium implants. A histometric analysis of the implanto-gingival junction around anloaded and loaded nonsubmerged implants in the canine mandible. J Periodontol. February 1997; Vol. 68; N²: 186 - 197.

4. P. Martínez Canut. Alargamiento de corona dentaria. I: bases anatómicas aplicadas. Periodoncia. 1996, Julio Septiembre; Vol. 6; $N^{\circ}$ 3: $153-163$.

5. Hyman Smukler, Mohammed Chaibi. Consideraciones periodontales y dentales en el procedimiento de alargamiento coronal: una base racional para su tratamiento. Revista Internacional de Odontología Restauradora y Periodoncia. 1997; Vol. 1; N 5: 441 - 453.

6. Jan Lindhe. Clinical periodontology and implant dentistry. Editorial Munksgaard. $3^{a}$ Edición. 1998. Capítulo 1: Anatomy of the periodontium: 19-68.

7. Antonio Bascones Martínez. Periodoncia. Diagnóstico y tratamiento de la enfermedad periodontal. Ed. Avances.

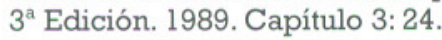

8. William Becker, Clifford Ochsenbein y Burton E. Becker. Crown lengthening: the periodontal-restorative connection. Compendium. March 1998; Vol. 19; No 3: 239-254.

9. Coslet J G y cols. Diagnosis and classification of delayed passive eruption of the dentogingival junction in the adult. Alpha Omegan. 1977; 3: 24 - 28 en Dolt III A H, William J. Altered passive eruption: An etiology of short clinical crowns. Quintessence International. 1997; Vol 28; $6: 363-372$.

10. J. S.Vacek, M. E. Gher, D. A. Assad, A. C. Richardson y L. I. Giambarresi. The dimensions of the human dentogingival junction. The International Journal of Periodontics \& Restorative Dentistry. 1994; Vol. 14; N²: 154-165.

11. Seibert J, Lindhe J. Textbook of clinical periodontology. Editorial Munksgaard. 2a Edición.1989. Capítulo 19: Esthetics and periodontal therapy: $477-514$.

12. Olsson M, Lindhe J. Periodontal characteristics in individuals with varying form of the upper central incisors. J Clin Peridontol. 1991; Vol 18: 78-82.

13. G Calsina Gomis. Cómo conseguir un periodonto sano y estable para prótesis fija. Desde http://www.infomed.es/uvd/periodoncia/artsel-1/artsell.html hasta http://www.infomes.es/uvd/periodoncia/artsel-1/artsel6.html. 1997, Octubre.

14. De Waal H, Castellucci G. The Importance Of Restorative Margin Placement To The Biologic Width And Periodontal Health. Part I. Int J Periodont Rest Dent. 1993; Vol. 13; $\mathrm{N}^{\circ}$ 5: 460-471.

15. M. Davarpanah; C. Jansen; F. M. A. Vidjak; D. Étienne; M. Kebir; H. Martínez. Consideraciones restauradoras y periodontales de coronas clínicas cortas. Revista Internacional de Odontología Restauradora y Periodoncia. 1998; Vol. 2; N 5: 400-409.

16. Richard D. Trushkowsky. Esthetic, biologic and restorative considerations in coronal reattachment for a fractured tooth: a clinical report. The Journal of Prosthetic Dentistry. 1998. Vol. 79; $\mathrm{N}^{\circ}$ 2: 115-119.

17. A H Dolt III, J W Robbins. Altered passive eruption: An etiology of short clinical crowns. Quintessence International. 1997; Vol 28; 6: $363-372$.

18. Jan Lindhe. Clinical periodontology and implant dentistry. Editorial Munksgaard. $3^{a}$ Edición. 1998. Capítulo 21: Esthetics in periodontal therapy: $647-658$.

19. Robert A Levine. Forced eruption in the esthetic zone. Compendium. August 1997; Vol. 18; N 8: $795-803$.

20. Ramfjord SE. Periodontal considerations of operative dentistry. Oper Dent 1988; 13: 144 - 159. 